\title{
Synthesis of Triarylamines via Sequential CN Bond Formation
}

DOI:

10.1021/acs.joc.7b01778

\section{Document Version}

Accepted author manuscript

Link to publication record in Manchester Research Explorer

\section{Citation for published version (APA):}

Modha, S., Popescu, M., \& Greaney, M. (2017). Synthesis of Triarylamines via Sequential CN Bond Formation. The Journal of organic chemistry, 82, 11933-11938. https://doi.org/10.1021/acs.joc.7b01778

\section{Published in:}

The Journal of organic chemistry

\section{Citing this paper}

Please note that where the full-text provided on Manchester Research Explorer is the Author Accepted Manuscript or Proof version this may differ from the final Published version. If citing, it is advised that you check and use the publisher's definitive version.

\section{General rights}

Copyright and moral rights for the publications made accessible in the Research Explorer are retained by the authors and/or other copyright owners and it is a condition of accessing publications that users recognise and abide by the legal requirements associated with these rights.

\section{Takedown policy}

If you believe that this document breaches copyright please refer to the University of Manchester's Takedown Procedures [http://man.ac.uk/04Y6Bo] or contact uml.scholarlycommunications@manchester.ac.uk providing relevant details, so we can investigate your claim.

\section{OPEN ACCESS}




\title{
Synthesis of Triarylamines via Sequential C-N Bond Formation
}

\author{
Sachin G. Modha, Mihai V. Popescu and Michael F. Greaney*
}

School of Chemistry, University of Manchester, Oxford Rd, Manchester M13 9PL, UK

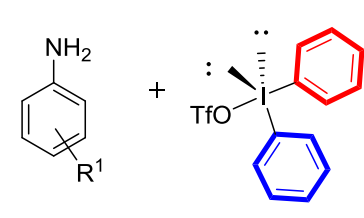

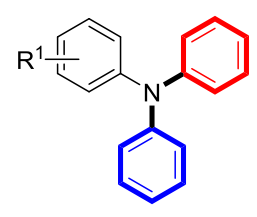

15 examples

yields up to $75 \%$

\begin{abstract}
A one-pot domino N-arylation protocol is described using diaryliodonium reagents under copper catalysis. The reaction uses both aryl groups of the diaryliodonium reagent to generate triarylamines starting from simple anilines, representing an atom-economical preparation of an important class of organic material building blocks.
\end{abstract}

Diaryliodonium reagents, discovered in $1894,{ }^{1}$ have seen extensive development as electrophilic arylating agents for carbon and heteroatom nucleophiles. ${ }^{2}$ They exhibit an appealing combination of stability, being crystalline solids that are easy to handle and store, and reactivity: Recent applications encompass aryl radical generation and utilization under photoredox catalysis, ${ }^{3}$ metal-free arylation, ${ }^{4}$ stereo-controlled arylation of double bonds, ${ }^{5}$ aryne generation, ${ }^{6}$ and extensive metal-catalyzed arylation processes. ${ }^{7}$ Despite this extremely versatile portfolio of reactivity, almost all of these applications share the common drawback of generating at least one equivalent of an iodobenzene as a waste product $-c a$. $50 \%$ of the atomic mass of the iodonium reagent, depending on the counterion. Whilst the iodobenzene can in principle be separated from the product mixture and recycled, this poor atom economy is clearly an obstacle to wider application of these reagents on scale.

\section{Scheme 1. Atom economical use of diaryliodonium reagents}

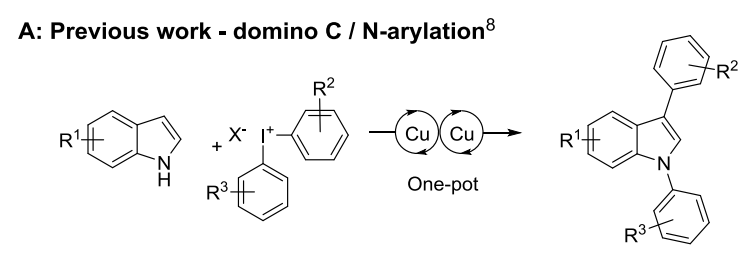

B: Domino borylation / Suzuki-coupling (Muniz) ${ }^{9}$

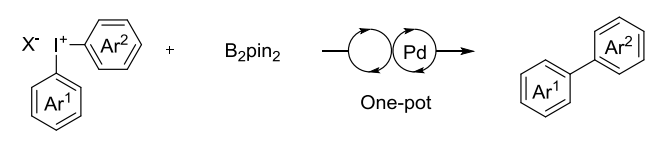

C: Domino S-arylation (Jiang) ${ }^{10}$

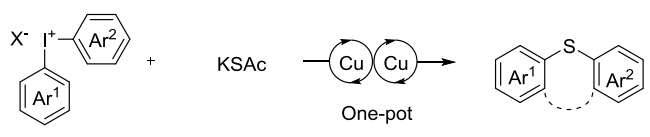

D: This Work - domino N-Arylation
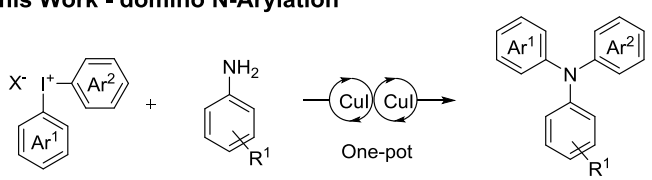

We have addressed this atom economy issue by designing domino processes that can capture the aryl iodide side product in a second arylation step in situ (Scheme1A) ${ }^{8}$ Copper-catalyzed C-H 
arylation of indole with a diaryliodonium salt, for example, can be followed by $\mathrm{N}$-arylation with the liberated aryl iodide to yield di-arylated products. This concept of domino arylation was subsequently demonstrated by Muñiz and co-workers who reported borylation / Suzuki-Miyaura coupling of diaryliodoniums and diboron reagents, ${ }^{9}$ and further developed by Jiang and co-workers, who described double arylation of a sulfur salt under copper catalysis to produce diarylsulfides from diaryliodoniums (Scheme 1B and C). ${ }^{10}$ Similar double-functionalization ideas have been explored with $\operatorname{ArI}(\mathrm{OAc})_{2}$ by Dauban and co-workers, and alkynyl iodine(III) derivatives by the Yoshikai and Waser groups. ${ }^{11}$ Collectively, these reports demonstrate that aryliodine(III) reagents can be used as powerful arene synthons in a variety of contexts, without having to sacrifice atom-economy. Indeed, by offering the possibility of two transformations from a single reagent, they create new opportunities for streamlined reaction design.

We were interested in further exploring the scope of domino applications of iodonium reagents, and chose to investigate the synthesis of triarylamines (Scheme 1D). Triarylamines are important building blocks in organic electronics, where their electron donor properties and characteristic propeller shapes have seen extensive application in the field of photovoltaics. ${ }^{12}$ They are commonly accessed through variants of the Ullman coupling, using excess aryl halide and high temperatures to drive reaction to completion. Synthesis using a single equivalent of iodonium reagent would present an alternative, and very direct route, to their preparation. The groups of Nachtsheim and Wen have described the domino assembly of $\mathrm{N}$-aryl carbazoles through reaction of anilines with cyclic iodonium salts, but the synthesis of triarylamines remains unexplored..$^{13}$

Our initial trial reactions examined the coupling of $p$-toluidine, 1a, with one equivalent of diphenyliodonium triflate, 2a, to give either the mono or di-phenylated products $\mathbf{3 a}$ and $\mathbf{5 a}$ (Table 1), using toluene as the solvent of choice. It was apparent that monophenylation was straightforward under both copper-catalysis at room temperature and metal-free conditions at $130{ }^{\circ} \mathrm{C},{ }^{\mathbf{1 4}}$ but there was no trace of the diphenylated product in either case (Entries 1 and 2). Despite the good yields of 3a, a small amount of starting $p$-toluidine remained in each case, which would potentially complicate the second N-arylation. Switching to the organic base 2,6-di-tert-butylpyridine (DTBPY) gave complete conversion and improved the yield up to $86 \%$ (entry 3), and catalyst loading could be decreased to 2 mol\% without loss of yield or conversion (entry 4).

With conditions established for high conversions of one equivalent of iodonium reagent in the first $\mathrm{N}$ arylation, we turned to the two-step one-pot sequence. Copper-catalyzed $\mathrm{N}$-arylation of weakly nucleophilic diarylamines usually requires a strong base and ligand system - we initially trialed an addition of $\mathrm{CuI}(10 \mathrm{~mol} \%)$, bis(2,6-diisopropylphenyl)diaza-butadiene (DAB) ${ }^{15}(12 \mathrm{~mol} \%)$ and $\mathrm{KO} t \mathrm{Bu}$ (2.2 equiv) to the reaction following initial $\mathrm{N}$-phenylation, and stirring at $120^{\circ} \mathrm{C}$ for $24 \mathrm{~h}$. The desired $N, N$-diphenyl-4-methylaniline (5a) could be isolated in 35\% yield, but significant amounts of unreacted 3a (42\%) were present (entry 5). Increased ligand loading did not improve the yield (entry 6 ), and a switch to Pd-catalysis for the second step was completely ineffective (entry 7). Using phenanthroline as ligand was initially unsuccessful when paired with $\mathrm{K}_{3} \mathrm{PO}_{4}$ (entry 8), but changing back to $\mathrm{KO} t \mathrm{Bu}$ in the presence of $10 \% \mathrm{CuI}$, gave good conversions to the product $\mathbf{5 a}$ (59\% yield over two steps) along with a small amount of $\mathbf{3 a}$ (Table 1, entry 10).

Having optimized conditions in hand for the one-pot double $\mathrm{N}$-arylation sequence, we examined the diphenylation of various aniline substrates. A variety of substituents $(o, m, p$-methyl, $o, p$-fluoro, $p$ chloro, $p$-methoxy and $m$-phenyl) were well-tolerated to give substituted triarylamines in good yields over two steps (Table 2). As for $p$-toluidine, small amounts of the mono-arylated product were isolated as side products in some cases (Table 2, entries 1, 5-7, 9). Variously substituted diaryliodonium triflates were employed next, to study the scope of the reagent with aniline. Iodonium reagents containing tert-butyl, fluoro, chloro, methoxy, and methyl reacted smoothly to give the desired triarylamines in good yields (Table 3 ), with a single equivalent of iodonium reagent being employed in each case. In terms of limitations, anilines containing strong electron withdrawing groups (e.g. $\left.p-\mathrm{NO}_{2}\right)$ were ineffective, being too deactivated to undergo the second arylation step. Attempts at selective aryl transfer using unsymmetrical diaryliodoniums were also unsuccessful under the current protocol, giving low yields of mixtures of $\mathrm{N}$-arylated material in the first step. 
Table 1. Reaction optimization ${ }^{a}$
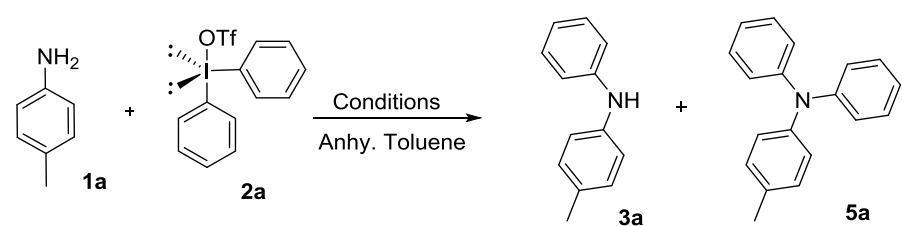

\begin{tabular}{|c|c|c|c|c|c|}
\hline entry & catalyst $(\mathrm{mol} \%) /$ ligand $(\mathrm{mol} \%)$ & base (equiv) & temp $\left({ }^{\circ} \mathrm{C}\right)$ & time $(\mathrm{h})$ & $\begin{array}{l}\text { yield }(\%) \\
(\mathbf{3 a}: 5 a)\end{array}$ \\
\hline 1 & $\mathrm{CuI}(10)$ & $\mathrm{K}_{2} \mathrm{CO}_{3}(1.5)$ & $\mathrm{rt}$ & 24 & $72: 0^{b}$ \\
\hline 2 & - & - & 130 & 24 & $68: 0^{b}$ \\
\hline 3 & $\mathrm{CuI}(10)$ & DTBPY (1.1) & $\mathrm{rt}$ & 24 & $86: 0$ \\
\hline 4 & $\mathrm{CuI}(2)$ & DTBPY (1.03) & $\mathrm{rt}$ & 12 & $87: 0$ \\
\hline 5 & $\begin{array}{l}\mathrm{CuI}(2) \text {; then } \\
\mathrm{CuI}(10) \text { and DAB (12) }\end{array}$ & $\begin{array}{l}\mathrm{DTBPY}(1.03) \text {; then } \\
\mathrm{KO} t \mathrm{Bu}(2.2)\end{array}$ & $\mathrm{rt}$; then 120 & $12 ;$ then 24 & $42: 35$ \\
\hline 6 & $\begin{array}{l}\mathrm{CuI}(2) \text {; then } \\
\mathrm{CuI}(10) \text { and } \mathrm{DAB}(30)\end{array}$ & $\begin{array}{l}\mathrm{DTBPY}(1.03) \text {; then } \\
\mathrm{KO} t \mathrm{Bu}(4)\end{array}$ & rt; then 120 & $12 ;$ then 24 & $35: 32$ \\
\hline 7 & $\begin{array}{l}\mathrm{CuI}(2) \text {; then } \\
\mathrm{Pd}(\mathrm{OAc})_{2}(4) \text { and } \mathrm{BINAP}(8)\end{array}$ & $\begin{array}{l}\mathrm{DTBPY}(1.03) \text {; then } \\
\mathrm{KO} t \mathrm{Bu}(2.2)\end{array}$ & $\mathrm{rt}$; then 120 & $12 ;$ then 24 & 62:traces \\
\hline 8 & $\begin{array}{l}\text { CuI (2); then } \\
\text { CuI (5) and 1,10-phenanthroline (6) }\end{array}$ & $\begin{array}{l}\text { DTBPY (1.03); then } \\
\mathrm{K}_{3} \mathrm{PO}_{4}(2.5)\end{array}$ & rt; then 120 & $12 ;$ then 24 & 56 :traces \\
\hline 9 & $\begin{array}{l}\mathrm{CuI}(2) \text {; then } \\
\mathrm{CuI}(5) \text { and } 1,10 \text {-phenanthroline (6) }\end{array}$ & $\begin{array}{l}\mathrm{DTBPY}(1.03) \text {; then } \\
\mathrm{KO} t \mathrm{Bu}(2.5)\end{array}$ & $\mathrm{rt}$; then 120 & $12 ;$ then 24 & $52: 15$ \\
\hline 10 & $\begin{array}{l}\mathrm{CuI}(2) \text {; then } \\
\mathrm{CuI}(10) \text { and } 1,10 \text {-phenanthroline (30) }\end{array}$ & $\begin{array}{l}\mathrm{DTBPY}(1.03) ; \text { then } \\
\mathrm{KO} t \mathrm{Bu}(4.5)\end{array}$ & $\mathrm{rt}$; then 120 & $12 ;$ then 24 & $15: 59$ \\
\hline
\end{tabular}

${ }^{a}$ Unless otherwise noted, all reaction were run with $\mathbf{1 a}(0.3 \mathrm{mmol})$ and $\mathbf{2 a}(1.02$ equiv) in a crimped cap glass vial under an inert atmosphere. ${ }^{b}$ Incomplete conversion. 
Table 2. Scope of aniline substrate ${ }^{a}$

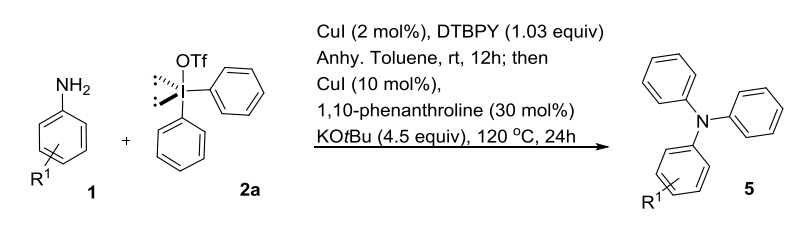

Table 3. Scope of diaryliodonium reagents ${ }^{a}$
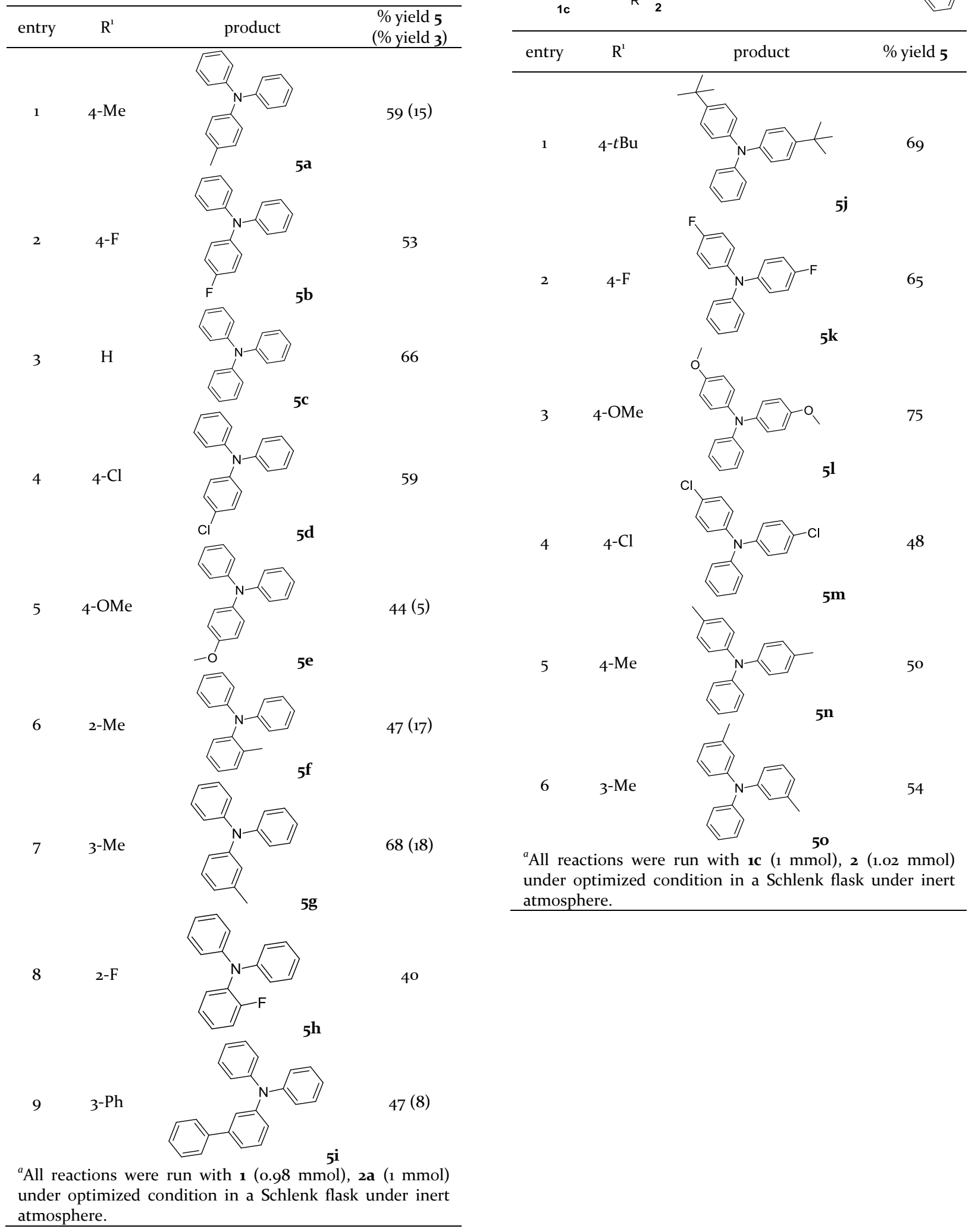

Cul (2 mol\%), DTBPY (1.03 equiv) Anhy. Toluene, rt, $12 \mathrm{~h}$; then Cul (10 mol\%),

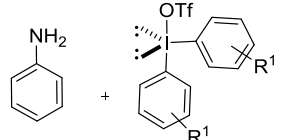

10-phenanthroline (30 mol\%) KOtBu (4.5 equiv), $120^{\circ} \mathrm{C}, 24 \mathrm{~h}$
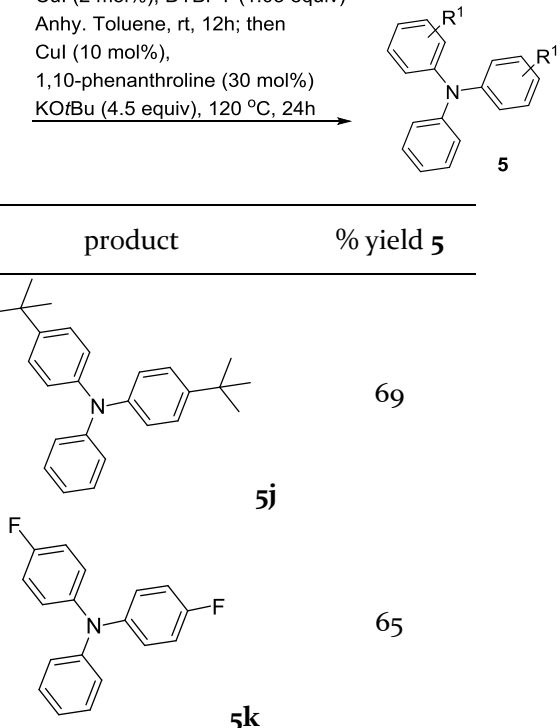

$2 \quad 4-\mathrm{F}$

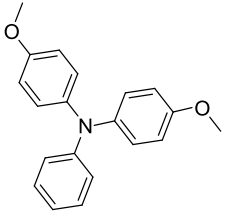

3 4-OMe<smiles>Clc1ccc(N(c2ccccc2)c2ccc(Cl)cc2)cc1</smiles>

51

$4 \quad 4-\mathrm{Cl}$

$5 \quad 4-\mathrm{Me}$

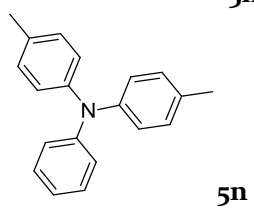

$6 \quad 3-\mathrm{Me}$

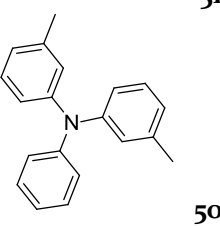

50

$5 n$

48

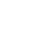

${ }^{a}$ All reactions were run with $1 \mathrm{c}(1 \mathrm{mmol}), 2$ (1.02 $\left.\mathrm{mmol}\right)$ under optimized condition in a Schlenk flask under inert atmosphere. 
A mechanistic pathway for the two step process is set out in Scheme 2, and likely involves a switch in mechanism between the first and second arylation steps. Whilst initial aniline arylation with the reactive diaryliodonium salt 2 proceeds through familiar $\mathrm{Cu}$ (I) / $\mathrm{Cu}$ (III) oxidative addition / reductive elimination processes,${ }^{16}$ Shyu and co-workers have implicated an electron transfer pathway for the second arylation of $\mathrm{N}$-arylaniline $\mathbf{3}$ under $\mathrm{KO} t \mathrm{Bu}$ and $\mathrm{CuI} /$ phenanthroline conditions. ${ }^{17}$ Electron transfer from the potassium complex of phenanthroline and diarylamine $\mathbf{D}$ ' to aryl iodide $\mathbf{4 a}$ generates an aryl radical which can be trapped with the $\mathrm{Cu}(\mathrm{I})$-phenanthroline diarylamine species B'. One electron oxidation by radical cation $\mathbf{E}$ ' then enables reductive elimination to furnish the triarylamine $5 \mathbf{a}$ and regenerate a $\mathrm{Cu}(\mathrm{I})$-phenanthroline species to continue the catalytic cycle.

Scheme 2. Mechanistic pathway for one-pot double $N$-arylation.

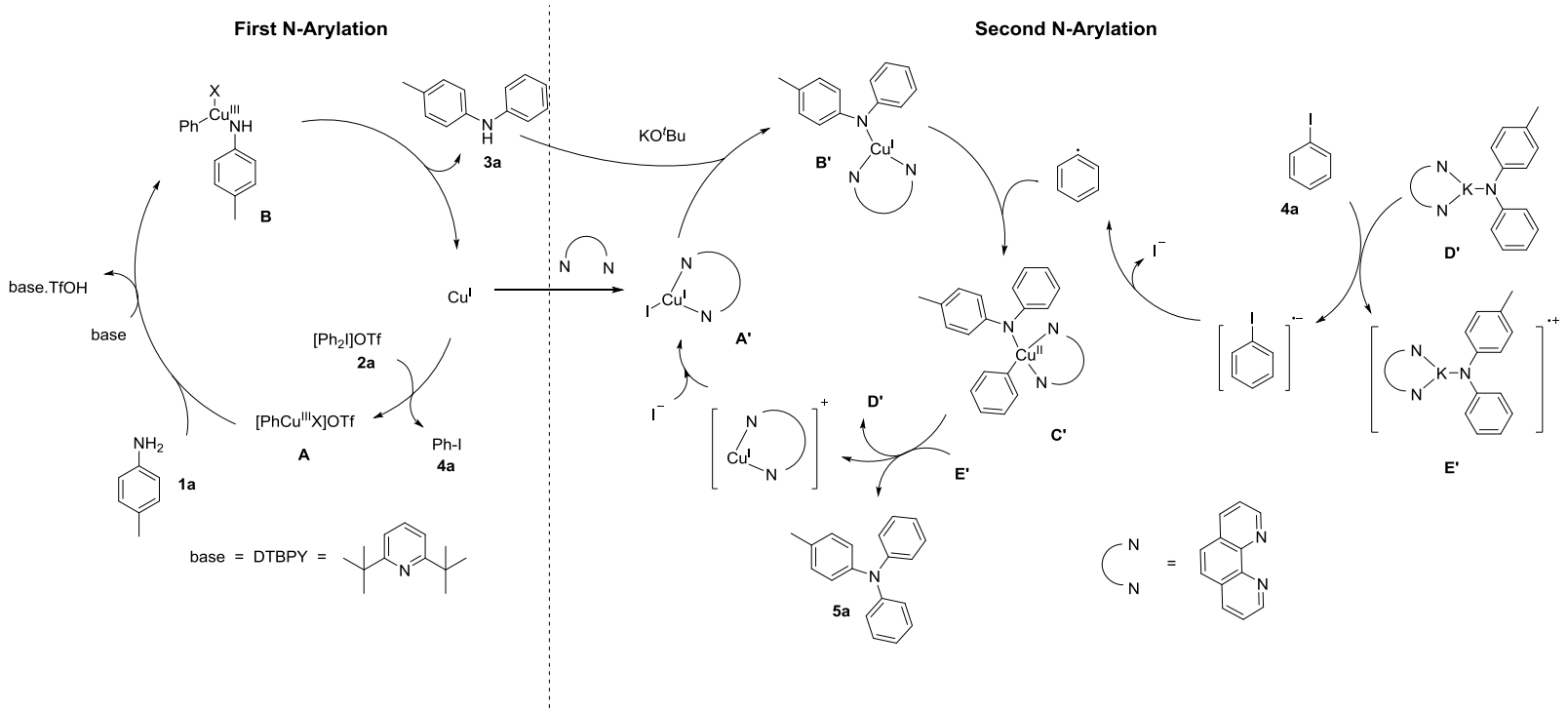

In summary, we have developed a copper-catalyzed, one-pot, domino $N$-arylation sequence to generate triarylamines starting from simple anilines. The protocol uses both aryl groups of diaryliodonium reagents, from a single equivalent of reagent, and a common $\mathrm{CuI}$ catalyst. Further atom-economical transformations of aryliodine (III) reagents are under investigation in our laboratory.

\section{EXPERIMENTAL}

General Information. Nuclear Magnetic Resonance (NMR) spectra were recorded on 500 or 400 $\mathrm{MHz}$ Bruker NMR spectrometers in $\mathrm{CDCl}_{3}$ at $298 \mathrm{~K}$ (unless stated otherwise). All chemical shift values are reported in parts per million (ppm) relative to the solvent signal (1H NMR: $\delta=7.26 \mathrm{ppm}$. 13C NMR: $\delta=77.16 \mathrm{ppm})$ with coupling constant $(J)$ values reported in Hz. The notation of signals is: Proton: $\delta$ chemical shift in ppm (number of protons, multiplicity, $J$ value(s), proton assignment). Carbon: $\delta$ chemical shift in ppm. Fluorine: $\delta$ chemical shift in ppm (Fluorine assignment). Splitting patterns are assigned $\mathrm{s}=$ singlet, $\mathrm{b}=$ broad, $\mathrm{d}=$ doublet, $\mathrm{td}=$ triplet of doublet, $\mathrm{dt}=$ doublet of triplet, $\mathrm{t}$ $=$ triplet, $\mathrm{q}=$ quartet, $\mathrm{bs}=$ broad singlet. Reactions were carried out under $\mathrm{N}_{2}$ using pre-dried glassware . All reaction were carried out in Schlenk flask and heated in oil baths with a thermocouple temperature control. Toluene, THF and dichloromethane were freshly distilled over sodium or calcium hydride and stored under $\mathrm{N}_{2}$. Other solvents, unless otherwise stated, were purchased in reagent grade or anhydrous quality and used as received. Reagents were either purchased directly from commercial suppliers or 
prepared according to literature procedures. TLC: Macherey-Nagel, TLC plates Alugram® Sil G/UV254. Detection under UV light at $254 \mathrm{~nm}$. Chromatography: Separations were carried out on Biotage $^{\circledR}$ Isolera Four flash chromatography instrument, with dryloading on Biotage SNAP Ultra columns using ethyl acetate / hexane (5 / 95 - 20 / 80 for triaryl amines, rising to 70 / 30 for elution of diarylamines when they were present). High Resolution Mass Spectrometry (HRMS) were recorded on Thermo Finnigan MAT95XP (ion trap). Melting points were determined using a Buchi M565 melting point apparatus. Iodonium salts $\mathbf{2 a - g}$ were prepared according to literature procedures. ${ }^{18}$

Representative procedure for domino $\mathrm{C}-\mathrm{N}$ bond formation: 4-Methyl- $N, N$-diphenylaniline (5a). To an oven dried $25 \mathrm{~mL}$ Schlenk flask containing a stir bar was added the 4-methylaniline (1a) (105 mg, $0.98 \mathrm{mmol}, 1.00$ equiv), CuI (3.8 $\mathrm{mg}, 0.02 \mathrm{mmol}, 2 \mathrm{~mol} \%$ ) and diphenyliodonium triflate (2a) (430 mg, $1 \mathrm{mmol}, 1.02$ equiv). The Schlenk flask was sealed with a glass stopper. A vacuum / $\mathrm{N}_{2}$ cycle was applied three times to the flask in order to ensure the removal of air. To this flask was added 2,6-di-tert-butylpyridine ( $227 \mu \mathrm{L}, 1.0094 \mathrm{mmol}, 1.03$ equiv) via glass syringe ( $250 \mu \mathrm{L}$ capacity) and dry Toluene $(10 \mathrm{~mL})$ via disposable syringe. The reaction mixture was allowed to stir at room temperature for $12 \mathrm{~h}$. After confirming completion of the reaction by TLC (Hexane/diethyl ether 8/2); CuI (18.7 mg, $0.098 \mathrm{mmol}, 10 \mathrm{~mol} \%$ ), 1, 10-phenanthroline (53 mg, $0.294 \mathrm{mmol}, 30 \mathrm{~mol} \%$ ) and $\mathrm{KO}^{t} \mathrm{Bu}$ (495 mg, $4.41 \mathrm{mmol}, 4.5$ equiv) were added to the flask followed by $\mathrm{N}_{2}$ flush to ensure the inert atmosphere and sealed again with the glass stopper. The flask-stopper joint was sealed by taflon tape and secured with a clip. The reaction mixture was then allowed to stir at $120{ }^{\circ} \mathrm{C}$ for $24 \mathrm{~h}$. After confirming the product formation by TLC (Hexane/diethyl ether - 9/1) the reaction mixture was diluted with EtOAc $(100 \mathrm{~mL})$ and washed with water $(50 \mathrm{~mL})$ (In case of emulsion, it was passed through a celite plug). The organic layer was further washed with brine $(50 \mathrm{~mL})$ dried over $\mathrm{MgSO}_{4}$, filtered and evaporated under reduced pressure. The crude was purified by Biotage flash chromatography instrument to yield 4-methyl- $N, N$-diphenylaniline 5a (156 mg, 59\% yield) as a white solid; MP $72-73{ }^{\circ} \mathrm{C} ;{ }^{1} \mathrm{H}-\mathrm{NMR}\left(500 \mathrm{MHz}, \mathrm{CDCl}_{3}\right): \delta 7.24-7.20(\mathrm{~m}, 4 \mathrm{H}), 7.08-7.05(\mathrm{~m}, 6 \mathrm{H}), 7.02-$ $6.99(\mathrm{~m}, 2 \mathrm{H}), 6.97(\mathrm{tt}, J=7.3,1.1 \mathrm{~Hz}, 2 \mathrm{H}), 2.32(\mathrm{~s}, 3 \mathrm{H}) ;{ }^{13} \mathrm{C}-\mathrm{NMR}\left(126 \mathrm{MHz}, \mathrm{CDCl}_{3}\right): \delta 148.0,145.2$, 132.7, 129.9, 129.1, 124.9, 123.6, 122.2, 20.8; HR-MS (EI) $\mathrm{m} / \mathrm{z}$ calcd for $\mathrm{C}_{19} \mathrm{H}_{18} \mathrm{~N}[\mathrm{M}+\mathrm{H}]^{+} 260.1439$, found 260.1433; and 4-methyl- $N$-phenylaniline 3a $(27 \mathrm{mg}, 15 \%)$ as a white solid; MP 88-90 ${ }^{\circ} \mathrm{C} ;{ }^{1} \mathrm{H}$ NMR (500 MHz, $\left.\mathrm{CDCl}_{3}\right): \delta 7.28-7.24(\mathrm{~m}, 2 \mathrm{H}), 7.12-7.10(\mathrm{~m}, 2 \mathrm{H}), 7.04-7.02(\mathrm{~m}, 4 \mathrm{H}), 6.90$ (bt, $J$ $=7.3,1.0 \mathrm{~Hz}, 1 \mathrm{H}), 5.62(\mathrm{bs}, 1 \mathrm{H}), 2.33(\mathrm{~s}, 3 \mathrm{H}) ;{ }^{13} \mathrm{C}-\mathrm{NMR}\left(126 \mathrm{MHz}, \mathrm{CDCl}_{3}\right): \delta 143.9,140.3,130.9$, $129.8,129.3,120.3,118.9,116.8,20.7$; HR-MS (EI) $\mathrm{m} / z$ calcd for $\mathrm{C}_{13} \mathrm{H}_{14} \mathrm{~N}[\mathrm{M}+\mathrm{H}]^{+} 184.1126$, found 184.1128.

4-Fluoro- $\boldsymbol{N}, \boldsymbol{N}$-diphenylaniline (5b). White solid (137 mg, 53\%). MP 98-100 ${ }^{\circ} \mathrm{C} ;{ }^{1} \mathrm{H}-\mathrm{NMR}(400 \mathrm{MHz}$, $\left.\mathrm{CDCl}_{3}\right): \delta 7.27-7.23(\mathrm{~m}, 4 \mathrm{H}), 7.11-7.04(\mathrm{~m}, 6 \mathrm{H}), 7.03-6.96(\mathrm{~m}, 4 \mathrm{H}) ;{ }^{13} \mathrm{C}-\mathrm{NMR}(101 \mathrm{MHz}$, $\left.\mathrm{CDCl}_{3}\right): \delta 158.90(\mathrm{~d}, J=243 \mathrm{~Hz}), 147.8,143.8(\mathrm{~d}, J=2.8 \mathrm{~Hz}), 129.2,126.5(\mathrm{~d}, J=8.0 \mathrm{~Hz}), 123.5$, 122.5, $116.0(\mathrm{~d}, J=22.5 \mathrm{~Hz}) ;{ }^{19} \mathrm{~F}$ NMR $\left(376 \mathrm{MHz}, \mathrm{CDCl}_{3}\right):-119.7$ (s, $\left.1 \mathrm{~F}\right)$; HR-MS (EI) $\mathrm{m} / \mathrm{z}$ calcd for $\mathrm{C}_{18} \mathrm{H}_{15} \mathrm{FN}[\mathrm{M}+\mathrm{H}]^{+}$264.1189, found 264.1180. 
Triphenylamine (5c). White solid (158 mg, 66\%). MP 127-129 ${ }^{\circ} \mathrm{C} ;{ }^{1} \mathrm{H}-\mathrm{NMR}\left(400 \mathrm{MHz}, \mathrm{CDCl}_{3}\right): \delta$ $7.28-7.24(\mathrm{~m}, 6 \mathrm{H}), 7.13-7.09(\mathrm{~m}, 6 \mathrm{H}), 7.05-7.0(\mathrm{~m}, 3 \mathrm{H}) ;{ }^{13} \mathrm{C}-\mathrm{NMR}\left(101 \mathrm{MHz}, \mathrm{CDCl}_{3}\right): \delta 147.8$, 129.2, 124.1, 122.6; HR-MS (EI) $\mathrm{m} / z$ calcd for $\mathrm{C}_{18} \mathrm{H}_{16} \mathrm{~N}[\mathrm{M}+\mathrm{H}]^{+} 246.1283$, found 246.1277.

4-Chloro- $N, N$-diphenylaniline (5d). White solid (162 mg, 59\%). MP 108-109 ${ }^{\circ} \mathrm{C}$; ${ }^{1} \mathrm{H}-\mathrm{NMR}$ (500 $\left.\mathrm{MHz}, \mathrm{CDCl}_{3}\right): \delta 7.27-7.24(\mathrm{~m}, 4 \mathrm{H}), 7.20-7.17(\mathrm{~m}, 2 \mathrm{H}), 7.08-7.06(\mathrm{~m}, 4 \mathrm{H}), 7.03-6.99(\mathrm{~m}, 4 \mathrm{H})$; ${ }^{13} \mathrm{C}-\mathrm{NMR}\left(126 \mathrm{MHz}, \mathrm{CDCl}_{3}\right): \delta 147.4,146.5,129.3,129.2,127.3,124.9,124.3,123.1$; HR-MS (EI) $\mathrm{m} / z$ calcd for $\mathrm{C}_{18} \mathrm{H}_{15} \mathrm{ClN}[\mathrm{M}+\mathrm{H}]^{+} 280.0893$, found 280.0885 .

4-Methoxy- $N, N$-diphenylaniline (5e). White solid (120 mg, 44\%). MP 102-103 ${ }^{\circ} \mathrm{C}$; ${ }^{1} \mathrm{H}-\mathrm{NMR}(500$ $\left.\mathrm{MHz}, \mathrm{CDCl}_{3}\right): \delta 7.23-7.19(\mathrm{~m}, 4 \mathrm{H}), 7.08-7.06(\mathrm{~m}, 2 \mathrm{H}), 7.05-7.03(\mathrm{~m}, 4 \mathrm{H}), 6.94(\mathrm{tt}, J=7.3,1.1$ $\mathrm{Hz}, 2 \mathrm{H}) 6.86-6.83(\mathrm{~m}, 2 \mathrm{H}), 3.80(\mathrm{~s}, 3 \mathrm{H}) ;{ }^{13} \mathrm{C}-\mathrm{NMR}\left(101 \mathrm{MHz}, \mathrm{CDCl}_{3}\right): \delta 156.1,148.1,140.7,129.0$, 127.3, 122.8, 121.8, 114.7, 55.5; HR-MS (EI) $m / z$ calcd for $\mathrm{C}_{19} \mathrm{H}_{18} \mathrm{NO}[\mathrm{M}+\mathrm{H}]^{+} 276.1388$, found 276.1380; and 4-Methoxy- $N$-phenylaniline (3e). White solid (9 mg, 5\%) Data of this compound were in accordance with those reported in literature. ${ }^{14 \mathrm{~b}}$

2-Methyl- $N, N$-diphenylaniline (5f). White solid $(120 \mathrm{mg}, 47 \%)$. MP $57-59{ }^{\circ} \mathrm{C}$; ${ }^{1} \mathrm{H}-\mathrm{NMR}(400 \mathrm{MHz}$, $\left.\mathrm{CDCl}_{3}\right): \delta 7.29-7.15(\mathrm{~m}, 8 \mathrm{H}), 7.02-6.98(\mathrm{~m}, 4 \mathrm{H}), 6.97-6.93(\mathrm{~m}, 2 \mathrm{H}), 2.07(\mathrm{~s}, 3 \mathrm{H}) ;{ }^{13} \mathrm{C}-\mathrm{NMR}(101$ $\left.\mathrm{MHz}, \mathrm{CDCl}_{3}\right): \delta 147.4,145.3,136.5,131.7,129.6,129.0,127.3,126.0,121.5,121.3$, 18.6; HR-MS (EI) $\mathrm{m} / z$ calcd for $\mathrm{C}_{19} \mathrm{H}_{18} \mathrm{~N}[\mathrm{M}+\mathrm{H}]^{+}$260.1439, found 260.1432; and 2-methyl- $\boldsymbol{N}$-phenylaniline (3f). White solid (30 mg, 17\%). Data of this compound were in accordance with those reported in literature. $^{19}$

3-Methyl- $\mathrm{N}, \mathrm{N}$-diphenylaniline (5g). White solid (173 mg, 68\%). MP 67-68 ${ }^{\circ} \mathrm{C} ;{ }^{1} \mathrm{H}-\mathrm{NMR}(400 \mathrm{MHz}$, $\left.\mathrm{CDCl}_{3}\right): \delta 7.28-7.24(\mathrm{~m}, 4 \mathrm{H}), 7.16(\mathrm{t}, J=7.7 \mathrm{~Hz}, 1 \mathrm{H}), 7.12-7.09(\mathrm{~m}, 4 \mathrm{H}), 7.04-7.0(\mathrm{~m}, 2 \mathrm{H}), 6.95-$ $6.90(\mathrm{~m}, 2 \mathrm{H}), 6.88-6.85(\mathrm{~m}, 1 \mathrm{H}), 2.29(\mathrm{~s}, 3 \mathrm{H}) ;{ }^{13} \mathrm{C}-\mathrm{NMR}\left(101 \mathrm{MHz}, \mathrm{CDCl}_{3}\right): \delta 147.9,147.7,139.0$, $129.1,129.0,124.9,124.0,123.7,122.4,121.5,21.4$; HR-MS (EI) $m / z$ calcd for $\mathrm{C}_{19} \mathrm{H}_{18} \mathrm{~N}[\mathrm{M}+\mathrm{H}]^{+}$ 260.1439, found 260.1434; and 3-methyl- $N$-phenylaniline (3g). Oil (32 mg, 18\%). Data of this compound were in acordance with those reported in literature. ${ }^{20}$

2-Fluoro- $N, \boldsymbol{N}$-diphenylaniline (5h). White solid (105 mg, 40\%). MP 70-72 ${ }^{\circ} \mathrm{C}$; ${ }^{1} \mathrm{H}-\mathrm{NMR}(500 \mathrm{MHz}$, $\left.\mathrm{CDCl}_{3}\right): \delta 7.28-7.24(\mathrm{~m}, 4 \mathrm{H}), 7.22-7.19(\mathrm{~m}, 1 \mathrm{H}), 7.17-7.09(\mathrm{~m}, 3 \mathrm{H}), 7.06-7.0(\mathrm{~m}, 6 \mathrm{H}) ;{ }^{13} \mathrm{C}-$ NMR (126 MHz, $\left.\mathrm{CDCl}_{3}\right): \delta 158.4(\mathrm{~d}, J=250 \mathrm{~Hz}), 147.2,134.6(\mathrm{~d}, J=10.3 \mathrm{~Hz}), 129.2(\mathrm{~d}, J=2 \mathrm{~Hz})$, 129.1, 126.0 (d, $J=7.9 \mathrm{~Hz}), 124.9$ (d, $J=3.7 \mathrm{~Hz}), 122.4,122.3,117.1(\mathrm{~d}, J=19.9 \mathrm{~Hz}) ;{ }^{19} \mathrm{~F}$ NMR $(376$ $\mathrm{MHz}, \mathrm{CDCl}_{3}$ ): -119.2 (m, 1F); HR-MS (EI) $\mathrm{m} / \mathrm{z}$ calcd for $\mathrm{C}_{18} \mathrm{H}_{15} \mathrm{FN}[\mathrm{M}+\mathrm{H}]^{+}$264.1189, found 264.1184.

$\boldsymbol{N}, \boldsymbol{N}$-diphenyl-[1,1'-biphenyl]-3-amine (5i). White solid (148 mg, 47\%). MP 153-155 ${ }^{\circ} \mathrm{C} ;{ }^{1} \mathrm{H}-\mathrm{NMR}$ $\left(500 \mathrm{MHz}, \mathrm{CDCl}_{3}\right): \delta 7.51-7.49(\mathrm{~m}, 2 \mathrm{H}), 7.40-7.37(\mathrm{~m}, 2 \mathrm{H}), 7.30(\mathrm{bt}, J=1.9 \mathrm{~Hz}, 1 \mathrm{H}), 7.3(\mathrm{t}, J=$ $7.6 \mathrm{~Hz}, 2 \mathrm{H}), 7.28-7.23(\mathrm{~m}, 5 \mathrm{H}), 7.15-7.13(\mathrm{~m}, 4 \mathrm{H}) 7.07-7.01(\mathrm{~m}, 3 \mathrm{H}) ;{ }^{13} \mathrm{C}-\mathrm{NMR}(101 \mathrm{MHz}$, 
$\left.\mathrm{CDCl}_{3}\right): \delta 148.3,147.7,142.3,140.9,129.5,129.2,128.6,127.3,127.1,124.2,122.9,122.8,122.7$, 121.5; HR-MS (EI) $\mathrm{m} / z$ calcd for $\mathrm{C}_{24} \mathrm{H}_{20} \mathrm{~N}[\mathrm{M}+\mathrm{H}]^{+} 322.15996$, found 322.1584 .

$N$-phenyl-[1,1'-biphenyl]-3-amine (3i). White solid (19 mg, 8\%). MP 86-88 ${ }^{\circ} \mathrm{C} ;{ }^{1} \mathrm{H}-\mathrm{NMR}(400 \mathrm{MHz}$, $\left.\mathrm{CDCl}_{3}\right): \delta 7.59-7.57(\mathrm{~m}, 2 \mathrm{H}), 7.45-7.42(\mathrm{~m}, 2 \mathrm{H}), 7.37-7.28(\mathrm{~m}, 5 \mathrm{H}), 7.18-7.12(\mathrm{~m}, 3 \mathrm{H}), 7.08-$ $7.06(\mathrm{~m}, 1 \mathrm{H}), 6.96(\mathrm{tt}, J=7.3,1.1 \mathrm{~Hz}, 1 \mathrm{H}), 5.78(\mathrm{bs}, 1 \mathrm{H}) ;{ }^{13} \mathrm{C}-\mathrm{NMR}\left(101 \mathrm{MHz}, \mathrm{CDCl}_{3}\right): \delta 143.5$, $142.9,142.5,141.1,129.7,129.4,128.7,127.4,127.1,121.2,120.0,118.0,116.6,116.4$; HR-MS (EI) $\mathrm{m} / \mathrm{z}$ calcd for $\mathrm{C}_{18} \mathrm{H}_{16} \mathrm{~N}[\mathrm{M}+\mathrm{H}]^{+} 246.1283$, found 246.1277 .

4-(tert-butyl)- $N$-(4-(tert-butyl)phenyl)- $N$-phenylaniline (5j). White solid (238 $\mathrm{mg}, 69 \%)$ MP $106-$ $108{ }^{\circ} \mathrm{C} ;{ }^{1} \mathrm{H}-\mathrm{NMR}\left(500 \mathrm{MHz}, \mathrm{CDCl}_{3}\right): \delta 7.26-7.20(\mathrm{~m}, 6 \mathrm{H}), 7.08-7.06(\mathrm{~m}, 2 \mathrm{H}), 7.03-7.0(\mathrm{~m}, 4 \mathrm{H})$, $6.95(\mathrm{tt}, J=7.3,1.1 \mathrm{~Hz}, 1 \mathrm{H}), 1.31(\mathrm{~s}, 18 \mathrm{H}) ;{ }^{13} \mathrm{C}-\mathrm{NMR}\left(126 \mathrm{MHz}, \mathrm{CDCl}_{3}\right): \delta 148.1,145.4,145.1,129.0$, 125.9, 123.7, 123.3, 121.8, 34.2, 31.4; HR-MS (EI) $\mathrm{m} / z$ calcd for $\mathrm{C}_{26} \mathrm{H}_{32} \mathrm{~N}[\mathrm{M}+\mathrm{H}]^{+}$358.2535, found 358.2529 .

4-fluoro- $N$-(4-fluorophenyl)- $N$-phenylaniline (5k). White solid $(183 \mathrm{mg}, 65 \%)$. MP $92-93{ }^{\circ} \mathrm{C} ;{ }^{1} \mathrm{H}$ NMR (500 MHz, $\left.\mathrm{CDCl}_{3}\right): \delta 7.27-7.24(\mathrm{~m}, 2 \mathrm{H}), 7.07-6.96(\mathrm{~m}, 11 \mathrm{H}) ;{ }^{13} \mathrm{C}-\mathrm{NMR}\left(126 \mathrm{MHz}, \mathrm{CDCl}_{3}\right)$ : $\delta 158.8(\mathrm{~d}, J=243 \mathrm{~Hz}), 148.0,143.9(\mathrm{~d}, J=2.8 \mathrm{~Hz}), 129.2,125.9(\mathrm{~d}, J=7.8 \mathrm{~Hz}), 122.8,122.3,116.1$ $(\mathrm{d}, J=22.5 \mathrm{~Hz}) ;{ }^{19} \mathrm{~F}$ NMR (376 MHz, $\left.\mathrm{CDCl}_{3}\right):-119.8(\mathrm{~m}, 2 \mathrm{~F})$; HR-MS (EI) $\mathrm{m} / z$ calcd for $\mathrm{C}_{18} \mathrm{H}_{14} \mathrm{~F}_{2} \mathrm{~N}$ $[\mathrm{M}+\mathrm{H}]^{+}$282.1094, found 282.1084 .

4-methoxy- $N$-(4-methoxyphenyl)- $N$-phenylaniline (5l). White solid (229 mg, 75\%). MP 104-106 ${ }^{\circ} \mathrm{C}$; ${ }^{1} \mathrm{H}-\mathrm{NMR}\left(500 \mathrm{MHz}, \mathrm{CDCl}_{3}\right): \delta 7.19-7.16(\mathrm{~m}, 2 \mathrm{H}), 7.06-7.03(\mathrm{~m}, 4 \mathrm{H}), 6.95-6.93(\mathrm{~m}, 2 \mathrm{H}), 6.88-$ $6.85(\mathrm{~m}, 1 \mathrm{H}), 6.84-6.81(\mathrm{~m}, 4 \mathrm{H}), 3.80(\mathrm{~s}, 6 \mathrm{H}) ;{ }^{13} \mathrm{C}-\mathrm{NMR}\left(126 \mathrm{MHz}, \mathrm{CDCl}_{3}\right): \delta$ 155.6, 148.7, 141.1, 128.9, 126.4, 120.9, 120.5, 114.6, 55.5; HR-MS (EI) $m / z$ calcd for $\mathrm{C}_{20} \mathrm{H}_{20} \mathrm{NO}_{2}[\mathrm{M}+\mathrm{H}]^{+} 306.1494$, found 306.1486 .

4-chloro- $N$-(4-chlorophenyl)- $N$-phenylaniline (5m). White solid $(150 \mathrm{mg}, 48 \%)$. MP $76-78{ }^{\circ} \mathrm{C} ;{ }^{1} \mathrm{H}$ NMR (500 MHz, $\left.\mathrm{CDCl}_{3}\right): \delta 7.28-7.25(\mathrm{~m}, 2 \mathrm{H}), 7.21-7.18(\mathrm{~m}, 4 \mathrm{H}), 7.06-7.03(\mathrm{~m}, 3 \mathrm{H}), 7.0-6.97$ (m, 4H); ${ }^{13} \mathrm{C}-\mathrm{NMR}\left(126 \mathrm{MHz}, \mathrm{CDCl}_{3}\right): \delta 147.0,146.1,129.5,129.3,127.8,125.0,124.4,123.5$; HRMS (EI) $m / z$ calcd for $\mathrm{C}_{18} \mathrm{H}_{14} \mathrm{Cl}_{2} \mathrm{~N}[\mathrm{M}+\mathrm{H}]^{+} 314.0503$, found 314.0498 .

4-methyl- $N$-phenyl- $N$-(p-tolyl)aniline (5n). White solid (137 mg, 50\%). MP 113-114 ${ }^{\circ} \mathrm{C} ;{ }^{1} \mathrm{H}-\mathrm{NMR}$ $\left(500 \mathrm{MHz}, \mathrm{CDCl}_{3}\right): \delta 7.22-7.18(\mathrm{~m}, 2 \mathrm{H}), 7.06-7.02(\mathrm{~m}, 6 \mathrm{H}), 7.0-6.98(\mathrm{~m}, 4 \mathrm{H}), 6.94$ (bt, $J=7.25$ $\mathrm{Hz}, 1 \mathrm{H}), 2.31(\mathrm{~s}, 6 \mathrm{H}) ;{ }^{13} \mathrm{C}-\mathrm{NMR}\left(101 \mathrm{MHz}, \mathrm{CDCl}_{3}\right): \delta 148.2$, 145.4, 132.3, 129.8, 129.0, 124.4, 122.9, 121.6, 20.8; HR-MS (EI) $\mathrm{m} / z$ calcd for $\mathrm{C}_{20} \mathrm{H}_{20} \mathrm{~N}[\mathrm{M}+\mathrm{H}]^{+} 274.1596$, found 274.1591.

3-methyl- $N$-phenyl- $N$-(m-tolyl)aniline (5o). White solid (147 mg, 54\%). MP $68-70{ }^{\circ} \mathrm{C} ;{ }^{1} \mathrm{H}-\mathrm{NMR}$ $\left(500 \mathrm{MHz}, \mathrm{CDCl}_{3}\right): \delta 7.27-7.24(\mathrm{~m}, 2 \mathrm{H}), 7.16(\mathrm{t}, J=7.8 \mathrm{~Hz}, 2 \mathrm{H}) 7.10-7.08(\mathrm{~m}, 2 \mathrm{H}), 7.01$ (bt, $J=$ $7.3 \mathrm{~Hz}, 1 \mathrm{H}), 6.94(\mathrm{bs}, 2 \mathrm{H}), 6.92-6.89(\mathrm{~m}, 2 \mathrm{H}), 6.87-6.84(\mathrm{~m}, 2 \mathrm{H}), 2.29(\mathrm{~s}, 6 \mathrm{H}) ;{ }^{13} \mathrm{C}-\mathrm{NMR}(126$ $\left.\mathrm{MHz}, \mathrm{CDCl}_{3}\right): \delta 148.0,147.8,139.0,129.1,128.9,124.9,123.9,123.5,122.3,121.4,21.4$; HR-MS (EI) $\mathrm{m} / z$ calcd for $\mathrm{C}_{20} \mathrm{H}_{20} \mathrm{~N}[\mathrm{M}+\mathrm{H}]^{+} 274.1596$, found 274.1588 . 


\section{ASSOCIATED CONTENT \\ AUTHOR INFORMATION}

\section{Corresponding Author}

*E-mail: Michael.Greaney@manchester.ac.uk

\section{ACKNOWLEDGMENT}

We thank the EPSRC for funding (postdoctoral support for S.G.M. and Leadership fellowship to M.F.G). We also thank the RSC for Undergraduate Research Bursary to M.V.P.

\section{Supporting information}

Copies of ${ }^{1} \mathrm{H}$ and ${ }^{13} \mathrm{C}$ NMR spectra are available free of charge on the ACS Publications website.

\section{REFERENCES}

${ }^{1}$ Hartmann, C.; Meyer, V. Ber. Dtsch. Chem. Ges. 1894, 27, 426.
${ }^{2}$ Reviews: a) Merritt, E. A.; Olofsson, B. Angew. Chem. Int. Ed. 2009, 48, 9052. b) Zhdankin, V. V.; Stang, P. J. Chem. Rev. 2008, 108, 5299. c) Aradi, K.; Tóth, B. L.; Tolnai, B. L.; Novák, Z. Synlett 2016, 27, 1456. d) Fananas-Mastral, M. Synthesis 2017, 49, 1905.

3 a) Neufeldt, S. R.; Sanford, M. S. Adv. Synth. Catal. 2012, 354, 3517. b) Fumagalli, G.; Boyd, S.; Greaney, M. F. Org. Lett. 2013, 15, 4398. c) Hopkinson, M. N.; Sahoo, B.; Glorius, F. Adv. Synth. Catal. 2014, 356, 2794. d) Jiang, H.; Cheng, Y.; Wang, R.; Zhang, Y.; Yu, S. Chem. Commun. 2014, 50, 6164.

${ }^{4}$ Selected examples: a) Lindstedt, E.; Ghosh, R.; Olofsson, B. Org. Lett. 2013, 15, 6070. b) Jalalian, N.; Ishikawa, E. E.; Silva, L. F.; Olofsson, B. Org. Lett. 2011, 13, 1552. c) Ackermann, L.; Dell'Acqua, M.; Fenner, S.; Vicente, R.; Sandmann, R.; Org. Lett. 2011, 13, 2358. d) Wen, J.; Zhang, R.-Y.; Chen, S.-Y.; Zhang, J.; Yu, X.-Q. J. Org. Chem. 2012, 77, 766. e) Gonda, Z.; Novak, Z. Chem. Eur. J. 2015, 21, 16801. f) Wu, S.-Y.; Ma, X.P.; Liang, C.; Mo, D.-L. J. Org. Chem. 2017, 82, 3232. g) Wang, Z.-X.; Shi, W.-M.; Bi, H.-Y.; Li, X.-H.; Su, G.F.; Mo, D.-L. J. Org. Chem. 2016, 81, 8014. h) Li, P.; Weng, Y.; Xu, X.; Cui, X. J. Org. Chem. 2016, 81, 3994.

5 a) Allen, A. E.; MacMillan, D. W. C. J. Am. Chem. Soc. 2011, 133, 4260. b) Bigot, W.; Williamson, A. E.; Gaunt, M. J. J. Am. Chem. Soc. 2011, 133, 13778. c) Harvey, J. S.; Simonovich, S. P.; Jamison, C. R.; MacMillan, D. W. C. J. Am. Chem. Soc. 2011, 133, 13782. d) Kieffer, M. E.; Chuang, K. V.; Reisman, S. E. J. Am. Chem. Soc. 2013, 135, 5557. e) Lukamto, D. H.; Gaunt, M. J. J. Am. Chem. Soc. 2017, 139, 9160.

${ }^{6}$ Sundalam, S. K.; Nilova, A.; Seidl, T. L.; Stuart, D. R. Angew. Chem. Int. Ed. 2016, 55, 8431.

${ }^{7}$ Selected recent examples: a) Phipps, R. J.; Gaunt, M. J. Science 2009, 323, 1593. b) Xiao, B.; Fu, Y.; Xu, J.; Gong, T.-J.; Dai, J.-J.; Yi, J.; Liu, L. J. Am. Chem. Soc. 2010, 132, 468. c) Fananas-Mastral, M; Feringa, B. L. J. Am. Chem. Soc. 2014, 136, 9894. d) Zhang, F.; Das, S.; Walkinshaw, A. J.; Casitas, A.; Taylor, M.; Suero, M. G.; Gaunt, M. J. J. Am. Chem. Soc. 2014, 136, 8851. e) Xie, H.; Yang, S.; Zhang, C.; Ding, M.; Liu, M.; Guo, J.; Zhang, F. J. Org. Chem. 2017, 82, 5250. f) Gemoets, H. P. L.; Laudadio, G.; Verstraete, K.; Hessel, V.; Noel, T. Angew. Chem. Int. Ed. 2017, 56, 7161.

${ }^{8}$ a) Modha, S. G.; Greaney, M. F. J. Am. Chem. Soc. 2015, 137, 1416. b) Teskey, C. J.; Sohel, S. M. A.; Bunting, D. L.; Modha, S. G.; Greaney, M. F. Angew. Chem. Int. Ed. 2017, 56, 5263.

${ }^{9}$ Mirrales, M.; Romero, R. M.; Fernández, E.; Muñiz, K. Chem. Commun. 2015, 51, 14068.

${ }^{10}$ Wang, M.; Wei, J.; Fan, Q.; Jiang, X. Chem. Commun. 2017, 53, 2918. 
${ }^{11}$ a) Lu, B.; Wu, J.; Yoshikai, N. J. Am. Chem. Soc. 2014, 136, 11598-11601. b) Hari, D. P.; Waser, J. J. Am. Chem. Soc. 2016, 138, 2190. c) Buendia, J.; Darses, B.; Dauban, P. Angew. Chem. Int. Ed. 2015, 54, 5697. d) Xie, F.; Zhang, Z.; Yu, X.; Tang, G.; Li, X. Angew. Chem. Int. Ed. 2015, 54, 7405. e) Buendia, J.; Grelier, G.; Darses, B.; Jarvis, A. G.; Taran, F.; Dauban, P. Angew. Chem. Int. Ed. 2016, 55, 7530.

${ }^{12}$ Wang, J.; Liu, K.; Ma, L.; Zhan, X. Chem. Rev. 2016, 116, 14675-14725.

${ }^{13}$ a) Zhu, D.; Chen, M.; Li, M.; Luo, B.; Zhao, Y.; Huang, P.; Xue, F; Rapposelli, S.; Pi, R.; Wen, S. Eur. J. Med. Chem. 2013, 68, 81. b) Riedmüller, S.; Nachtsheim, B. J. Beilstein J. Org. Chem. 2013, 9, 1202.

${ }^{14}$ a) Beringer, F. M.; Brierley, A.; Drexler, M.; Gindler, E. M.; Lumpkin, C. C. J. Am. Chem. Soc. 1953, 75, 2708. b) Carroll, M. A.; Wood, R. A. Tetrahedron 2007 63, 11349.

${ }^{15}$ Liu, Y.-H.; Chen, C.; Yang, L.-M. Tetrahedron Lett. 2006, 47, 9275.

${ }^{16}$ Kang, S.-K.; Lee, S.-H.; Lee, D. Synlett 2000, 1022.

${ }^{17}$ For a discussion of the mechanistic pathway in operation for $\mathrm{CuI} /$ phenanthroline and $\mathrm{KO} t$-Bu, see: a) Tseng, C.-K.; Lee, C.-R.; Tseng, M.-C.; Han, C.-C.; Shyu, S.-G. Dalton Trans. 2014, 43, 7020. b) Barham, J. P.; Coulthard, G.; Emery, K. J.; Doni, E.; Cumine, F.; Nocera,G.; John, M. P.; Berlouis, L. E. A.; McGuire, T.; Tuttle, T.; Murphy, J. A. J. Am. Chem. Soc. 2016, 138, 7402.

${ }^{18}$ a) Bielawski, M.; Zhu, M.; Olofsson, B. Adv. Synth. Catal. 2007, 349, 2610, b) Bielawski, M.; Aili, D.; Olofsson, B. J. Org. Chem. 2008, 73, 4602, c) Toh, Q. Y.; McNally, A.; Vera, S.; Erdmann, N.; Gaunt, M. J. J. Am. Chem. Soc. 2013, 135, 3772, d) Kuriyama, M.; Hamaguchi, N.; Onomura, O. Chem. Eur. J. 2012, 18, 1591.

${ }^{19}$ Mostafalu, R.; Kaboudin, B.; Kazemi, F.; Yokomatsu, T. RSC. Adv. 2014, 4, 49273.

${ }^{20}$ Anuradha; Kumari, S.; Pathak, D. D. Tetrahedron Lett. 2015, 56, 4135. 